\title{
ג2,6-Hyposialylation of c-Met abolishes cell motility of ST6Gal-I-knockdown HCT116 cells
}

\author{
Jin QIAN ${ }^{1}$, Cai-hua ZHU ${ }^{1}$, Shuai TANG ${ }^{2}$, Ai-jun SHEN ${ }^{1}$, Jing Al ${ }^{2}$, Jing $\mathrm{LI}^{2}$, Mei-yu GENG ${ }^{1,2, *}$, Jian DING ${ }^{1, *}$ \\ ${ }^{1}$ Division of Anti-tumor Pharmacology, Shanghai Institute of Materia Medica, Shanghai 201203, China; ${ }^{2}$ Department of Pharmacology, \\ Marine Drug and Food Institute, Ocean University of China, Qingdao 266003, China
}

\begin{abstract}
Aim: We aimed to investigate the potential modification of previously unrecognized surface glycoprotein(s) by $\alpha 2,6$-sialylation other than by integrins.

Methods: The expression of $\beta$-galactoside $\alpha 2,6$-sialyltransferase (ST6Gal-I) in the colon cancer cell line HCT116 was reduced by siRNA. The adhesion and Boyden chamber assay were used to detect the variation in cell motility. $\alpha 2,6-$ Sialylation proteins were detected with lectin affinity assay. The mRNA expression, protein expression and downstream signaling modulation with siRNA were detected using reverse transcription-polymerase chain reaction, flow cytometry analysis, and Western blot.

Results: In HCT116 cells, the knockdown of ST6Gal-I inhibited cell motility, but did not affect cell adhesion. This selectively altered cell migration was caused by the loss of $\alpha 2,6$-sialic acid structures on c-Met. Moreover, STAT3 was dephosphorylated at tyrosine 705 in ST6Gal-I-knockdown (ST6Gal-I-KD) HCT116 cells.

Conclusion: c-Met is the substrate of ST6Gal-I. The hyposialylation of c-Met can abolish cell motility in ST6Gal-I-KD HCT116 cells.
\end{abstract}

Keywords: cell motility; c-Met; hyposialylation; ST6Gal-I

Acta Pharmacologica Sinica (2009) 30: 1039-1045; doi: 10.1038/aps.2009.84; published online 1 June 2009

\section{Introduction}

Cell surface proteins in mammals are typically elaborated with a complex array of asparagine-linked (N-linked) glycans. Sialic acids are usually found at the non-reducing terminal position of these N-glycans. This terminal sialylation imparts a negative charge at physiological $\mathrm{pH}$ values and mediates many biological functions. Altered expression of certain sialic acid types or their linkages is closely associated with cellular adhesion, migration and metastasis in tumor cells ${ }^{[1,2]}$.

Overexpression of $\beta$-galactoside a2,6-sialyltransferase (ST6Gal-I) has been observed in numerous types of human tumors, and it results in the increase of N-glycans in the Sia6LacNAc stucture in various cancer cells ${ }^{[3]}$. Emerging evidences showed that hypersialylation of $\beta 1$-integrin stimulated both cell attachment and migration on collagen I and induced variation in cell motility ${ }^{[4,5]}$. However, hypersialylation of $\beta 1$-integrin could not explain all phenotypic events. We therefore hypothesized that ST6Gal-I could modify other surface glycoprotein(s), which might directly or in synergy with integrins be responsible for the biological behavior.

* To whom correspondence should be addressed. E-mail gengmy@ouc.edu.cn; suozhang@mail.shcnc.ac.cn Received 2009-04-10 Accepted 2009-05-04
In the present study, we aimed to investigate the potential modification of previously unrecognized surface glycoprotein(s) by ST6Gal-I. The expression of ST6Gal-I in the human HCT116 colon carcinoma cell line was transiently knocked down with siRNA. Finally, we demonstrated a functional role for ST6Gal-I-driven terminal sialylation of c-Met glycoprotein in colon cancer progression, which might be distinct from integrin-dependent cancer progression.

\section{Materials and methods}

Cell lines and culture conditions

The colon cancer adenocarcinoma cell line, HCT116, was obtained from the American Type Culture Collection (Manassas, VA, USA). Cells were propagated and maintained in McCoy's 5A Medium (Sigma, St Louis, MO, USA) containing $100 \mathrm{U} / \mathrm{mL}$ penicillin and supplemented with 10\% fetal bovine serum (FBS, Gibco, Grand Island, NY, USA). Cells were maintained at $37{ }^{\circ} \mathrm{C}$ under a humidified $95 \%$ and $5 \%(v / v)$ mixture of air and $\mathrm{CO}_{2}$.

Short interfering (si)RNA transfection siRNA duplexes (D3) 5'-AACTCTCAGTTGGTTACCACA-3' specific for the human ST6Gal-I gene sequences, as well as control nontargeting siRNA (NC), were purchased from 
Shanghai Genepharma Co Ltd (Shanghai, China). Briefly, HCT116 cells were grown to $75 \%$ confluence, exposed to siRNA $(100 \mathrm{nmol} / \mathrm{L})$ for $6 \mathrm{~h}$ in the presence of oligofectamine (Invitrogen, Carlsbad, CA, USA), and then incubated at $37^{\circ} \mathrm{C}$ for $24 \mathrm{~h}$ before use in assays.

\section{RNA extraction and reverse transcription-polymerase chain reaction (RT-PCR)}

Total RNA was extracted from transfected cells using TRIzol (Invitrogen) after cells had reached about $90 \%$ confluence. After RT, cDNA was amplified by PCR using Taq DNA polymerase, deoxynucleoside triphosphates and the indicated primers, as described previously ${ }^{[6]}$, and 30 cycles of amplification were performed. The PCR products $(10 \mu \mathrm{L})$ were analyzed by electrophoresis on $2 \%$ agarose gels. After being stained with ethidium bromide, gel images were obtained using Genesnap Version 6.0026 software (Syngene, Cambridge, England).

\section{ST6Gal-I activity detection}

The activity of ST6Gal-I was determined by an enzyme-linkedimmunosorbent assay (ELISA) using asialofetuin (Sigma) precoated plates. Equivalent amounts of cell lysates were loaded and cytidine-5'-monophospho-N-acetylneuraminic acid sodium salt (CMP-NeuAC, Sigma) was then added to initiate the reaction. After being washed and blocked, the sialylated fetuin was bound with SNA-biotin (Vector Lab, Burlingame, CA, USA), and probed with streptavidin-horseradish peroxidase. Finally, $100 \mu \mathrm{L}$ of solution $\left(0.03 \% \mathrm{H}_{2} \mathrm{O}_{2}, 2 \mathrm{mg} / \mathrm{mL}\right.$ o-phenylenediamine in citrate buffer $0.1 \mathrm{~mol} / \mathrm{L}, \mathrm{pH} 5.5)$ was added and the reaction was terminated with $\mathrm{H}_{2} \mathrm{SO}_{4}$. The absorbance at $492 \mathrm{~nm}$ was measured using a multiwell spectrophotometer (VERSAmax, Molecular Devices, Union City, CA, USA).

\section{Fluorescence activated cell sorting (FACS) analysis}

For analysis of the cell surface a2,6-sialic acid structure and c-Met protein, adherent HCT116 cells were trypsinized and placed in FACS blocking buffer at a concentration of $5 \times 10^{5}$ cells $/ \mathrm{mL}$. The cells were then incubated with $2 \mu \mathrm{g}$ SNA-biotin or $0.5 \mu \mathrm{g}$ c-Met antibody (R\&D, Minneapolis, MN, USA) for 30 min on ice, washed twice in blocking buffer, and then exposed to $0.25 \mu \mathrm{g}$ R-phycoerythrin (RPE)-conjugated streptavidin for an additional $30 \mathrm{~min}$. Labeled samples were analyzed by flow cytometry.

\section{Cell adhesion assay}

Adhesion assays were performed in 96-well plates pre-coated with collagen $(10 \mu \mathrm{g} / \mathrm{mL})$, fibronectin $(10 \mu \mathrm{g} / \mathrm{mL})$ or laminin $(20 \mu \mathrm{g} / \mathrm{mL})$ (BD, San Jose, CA, USA). After being blocked with $1 \%$ bovine serum albumin, $0.4 \times 10^{5}$ cells were seeded into each well and allowed to adhere at $37^{\circ} \mathrm{C}$ for $1 \mathrm{~h}$. Non-adherent cells were rinsed with phosphate-buffered saline (PBS); the remaining cells were fixed with $4 \%$ paraformaldehyde and then stained with $0.1 \%$ crystal violet for $15 \mathrm{~min}$. After being rinsed with water, the remaining cells were solubilized by acetic acid. Absorbance spectroscopy $(595 \mathrm{~nm})$ was used to quantify the number of substrate-adherent cells.

\section{Migration assay}

Transwell Boyden chambers (Costar, Bethesda, MD, USA) were coated with gelatin. After being soaked with serum-free $5 \mathrm{~A}$ medium, $2 \times 10^{5}$ transfected cells suspended in $5 \mathrm{~A}$ containing $2 \%$ FBS were loaded into the upper well. The lower compartment was filled with culture medium alone or medium containing $100 \mathrm{ng}$ hepatocyte growth factor (HGF, R\&D) and then incubated at $37^{\circ} \mathrm{C}$ for 30 or $48 \mathrm{~h}$. After the non-migratory cells were removed, the migrated cells were fixed and stained as previously described and counted.

\section{Preparation of total cell extracts and immunoblot analysis}

After transfection with siRNA for $24 \mathrm{~h}$, cells were harvested and resuspended in RIPA lysis buffer $(50 \mathrm{mmol} / \mathrm{L}$ Tris- $\mathrm{HCl}$, $150 \mathrm{mmol} / \mathrm{L} \mathrm{NaCl}, 1 \mathrm{mmol} / \mathrm{L}$ EDTA, 0.1\% SDS, 0.5\% deoxycholic acid, $0.02 \%$ sodium azide, $1 \% \mathrm{NP}-40,2.0 \mu \mathrm{g} / \mathrm{mL}$ aprotinin, $1 \mathrm{mmol} / \mathrm{L}$ phenylmethylsulfonylfluoride). The effects of HGF stimulation were detected by serum starving transfected cells for $24 \mathrm{~h}$ and then stimulating with $50 \mathrm{ng}$ HGF for $20 \mathrm{~min}$. Equivalent amounts of proteins were used for Tyr1349-MET, Erk1/2, phosphorylated-Erk1/2, AKT, phosphorylated-AKT, STAT3, Tyr705-STAT3, Ser727-STAT3 (Cell Signaling Technology, Beverly, MA, USA) and/or $\beta$-subunit-specific antibody for MET (Santa Cruz Biotech, Santa Cruz, CA, USA) immunoblot detection.

\section{Lectin affinity assays}

Lysates were centrifuged at $10000 \times g$ for $15 \mathrm{~min}$ at $4{ }^{\circ} \mathrm{C}$. One milligram of supernatant was incubated for $4 \mathrm{~h}$ at $4{ }^{\circ} \mathrm{C}$ with $6 \mu \mathrm{g}$ SNA. Streptavidin-agarose beads (Sigma) were then added and incubated for an additional $4 \mathrm{~h}$ at $4{ }^{\circ} \mathrm{C}$ with rotation. After being briefly centrifuged and washed, precipitated proteins were released from the bead complexes by boiling in sodium dodecyl sulfate-polyacrylamide gel electrophoresis (SDS-PAGE) sample buffer and analyzed directly by SDSPAGE and immunoblotting.

\section{FACS analysis}

For analysis of the cell surface a2,3-sialic acid structure, adherent HCT116 cells were trypsinized with trypsin-EDTA using standard methods and placed in FACS blocking buffer $(0.15 \mathrm{~mol} / \mathrm{L}$ PBS with $2 \%$ BSA $)$ at a concentration of $5 \times 10^{5}$ cells $/ \mathrm{mL}$. The cells were then incubated with $2 \mu \mathrm{g}$ MAAbiotin (Vector Lab, Burlingame, CA) for $30 \mathrm{~min}$ on ice, washed twice in blocking buffer, and then exposed to $0.25 \mu \mathrm{g}$ RPEconjugated streptavidin for an additional $30 \mathrm{~min}$. Labeled samples were analyzed by flow cytometry.

\section{5-bromo-2'-deoxyuridine (BrdU) assay}

DNA synthesis was monitored by measuring incorporation of the artificial thymidine nucleotide analog BrdU (Sigma, St Louis, MO, USA) into newly synthesized DNA. Cells $\left(1.5 \times 10^{5}\right.$ per well) were cultured in 6-well plates and transfected with siRNAs. Forty-eight hours after transfection, cells 
were refreshed with complete medium containing 100 $\mu \mathrm{mol} / \mathrm{L} \mathrm{BrdU}$ and incubated for an additional $30 \mathrm{~min}$ for BrdU incorporation. After BrdU incubation, cells were washed three times with PBS and fixed in $4 \%$ paraformaldehydePBS for $20 \mathrm{~min}$. Next, the fixed cells were permeabilized with $0.5 \%$ Triton X-100-PBS. After partial denaturation of the DNA with $2 \mathrm{~mol} / \mathrm{L} \mathrm{HCl}$, cells were incubated with anti-BrdU mouse monoclonal antibody (Santa Cruz Biotechnology, CA), at a 1:50 dilution for $1 \mathrm{~h}$. Cells were then washed with PBS three times and incubated with Alexa Fluor 633 anti-mouse antibody (Invitrogen, Carlsbad, CA, USA) at a 1:200 dilution for $1 \mathrm{~h}$. After three PBS washes, cells were counterstained with $4^{\prime}, 6^{\prime}$-diamidino-2-phenylindole for $5 \mathrm{~min}$ and analyzed using an Olympus DP70 digital microscope camera. Quantitative analysis of immunofluorescence data was carried out with Image-Pro Plus software.

\section{Results}

Knockdown of ST6Gal-I reduced endogenous ST6Gal-I expression in HCT116 cells

Cell surface sialylation of the metastasizing HCT116 cell line correlates with in vivo tumorigenicity ${ }^{[2]}$, and overexpression of ST6Gal-I has been implicated in increased cell motility. Here, we sought to determine the effect of in vitro knockdown of ST6Gal-I expression on the motility of HCT116 cells. We transiently transfected ST6Gal-I-targeting siRNA into HCT116 cells (D3) and verified that ST6Gal-I mRNA expression was reduced by RT-PCR detection in a time-dependent manner (Figure 1A).

The activity of ST6Gal-I in siRNA transfected HCT116 cells (D3) was compared with that in parental HCT116 cells (P) and HCT116 cells transfected with nonspecific siRNA (NC) by ELISA assay. After interference with ST6Gal-I-targeting siRNA, the a2,6-sialic acid decoration was significantly decreased in D3 cells (Figure 1B).

The a2,6-sialic acid structures on the cell surface were evaluated by FACS analysis. As expected, the a2,6-sialic acid structures on the D3 cell surface were remarkably reduced compared with those in P and NC cells (Figure 1C), whereas the a2,3-sialic acid structures were not affected (Figure S1). These results indicated that siRNA effectively reduced the expression of ST6Gal-I.

\section{Decreased expression of ST6Gal-I reduces cell migration but not cell adhesion}

To determine the effect of cell surface a2,6-sialylation on cell motility, we evaluated cell migration using a modified Boyden chamber assay with FBS as a chemoattractant. The migration of D3 cells was significantly less than that of $\mathrm{P}$ or NC cells (Figure 1D). The growth kinetics study showed that the reduction in ST6Gal-I had no effect on cell proliferation (Figure S2). These results indicate that the ST6Gal-I regulated cell motility but not cell growth.

We next examined the effects of ST6Gal-I siRNA on cell adhesion, specifically to three main extracellular matrix (ECM) components: laminin, fibronectin, and collagen. The ability of
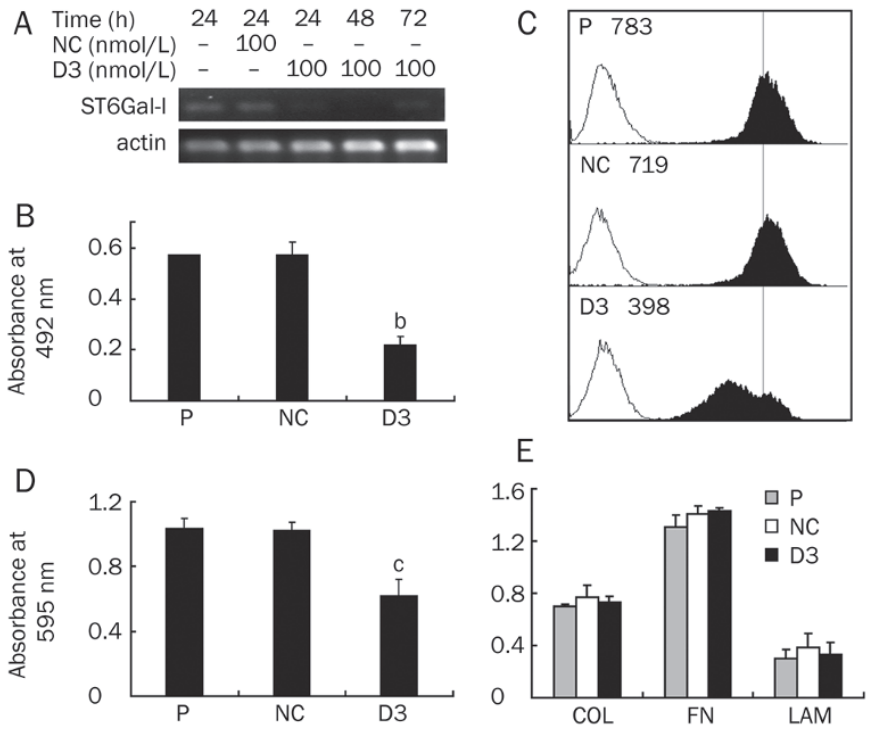

Figure 1. Cell motility is abolished in ST6Gal-I-KD HCT116 cells. (A) SiRNA-mediated knockdown of ST6Gal-I reduced ST6Gal-I mRNA expression in a time-dependent manner and was effective up to $72 \mathrm{~h}$. HCT116 cells were transfected with siRNA duplexes designed to target ST6Gal-I (D3); cells transfected with non-targeting siRNA (NC) and mock-transfected cells (P) served as controls. (B) D3 siRNA transfection decreased ST6Gal-I activity. After transfection, the cells were lysed and the same amounts of proteins were used in ELISAs. $\alpha 2,6$-sialic acid decoration of asialofetuin was detected with SNA. ${ }^{\mathrm{b}} \mathrm{P}<0.05$ vs NC cells. Columns, means of four independent experiments; bars, SD. (C) D3 siRNA transfection reduced cell surface expression of $\alpha 2,6$-sialic acid structures. P, NC, and D3 cells were detached using trypsin-EDTA, and $\alpha 2,6$-sialic acid structures were stained with SNA and analyzed by FACS. The data are representative of three independent experiments with similar results. (D) D3 siRNA transfection reduced HCT116 cell migration. The migratory capacity of cells was assessed using a Boyden transwell assay. After incubation for $30 \mathrm{~h}$, migrated cells on the lower surface of the filter were stained with crystal violet and solubilized by the addition of $100 \mu \mathrm{L} 10 \%$ acetic acid. ${ }^{\circ} P<0.01$ vs NC cells. Columns, means of four independent experiments; bars, SD. (E) D3 SiRNA transfection had no effect on cell adhesion. The relative numbers of attached P cells, NC cells, and D3 cells were assessed by staining with crystal violet, and absorbance was determined at $595 \mathrm{~nm}$. Columns, means of three independent experiments performed in triplicate; bars, SD. COL: collagen. FN: fibronectin. LAM: laminin.

D3 cells to attach to these three components was similar to that of $P$ and NC cells (Figure 1E), suggesting that downregulation of ST6Gal-I expression had no effect on cell adhesion.

\section{c-Met is hyposialylated in ST6Gal-I-KD HCT116 cells}

It has been postulated that changes in sialic acid structure on membrane glycoconjugates can modulate the adhesion of cancer cells to ECM components in an integrin-dependent manner ${ }^{[7-10]}$. Because we did not find any change in ST6GalI-KD HCT116 cell adhesion, we suggest that integrins alone cannot fully explain this observation. Sialylation of membrane glycoproteins other than integrins might be responsible for the ST6Gal-I-dependent motility phenotype in HCT116 cells. To identify the related membrane glycoproteins in the regulation 


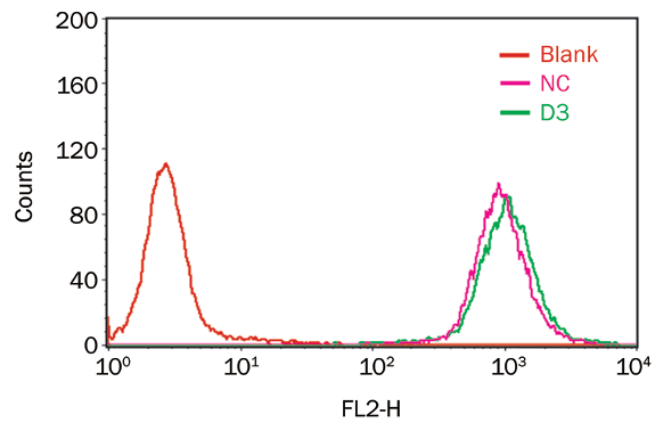

Figure S1. D3 siRNA transfection does not affect cell surface expression of $\alpha 2,3$-sialic acid structures. Adherent D3, NC, and P cells were first released by trypsinization and then labeled with $2 \mu \mathrm{g}$ MAA-biotin. After incubation with $0.25 \mu \mathrm{g}$ RPE-conjugated streptavidin, labeled cells were subjected to FACS analysis. The data are representative of three independent experiments with similar results.

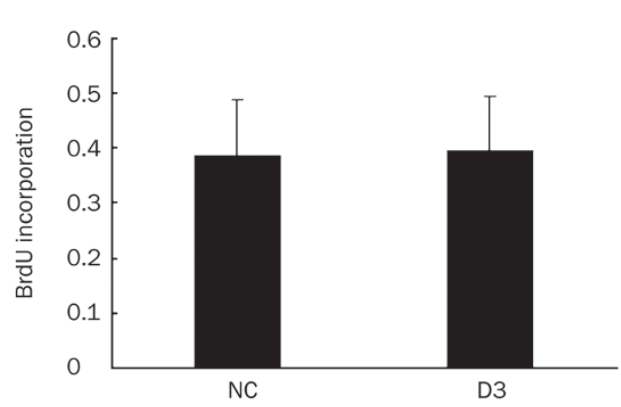

Figure S2. ST6Gal-I abolition did not affect growth kinetics in HCT116 cells. DNA synthesis in NC and D3 cells was monitored by measuring the incorporation of the artificial thymidine nucleotide analog BrdU into newly synthesized DNA, as described in Materials and Methods.

of cell motility, we precipitated a2,6-sialylated glycoproteins using SNA and then examined the glycoproteins of interest, c-Met and CXCR4, by immunoblotting. Remarkably, c-Met was clearly present in SNA precipitates prepared from cells with normal ST6Gal-I expression (P and NC), whereas little c-Met was present in ST6Gal-I-KD HCT116 cells (D3) (Figure 2A). In contrast, CXCR4 was not co-precipitated by SNA (data not shown).

To further examine the possibility that the decreased cell motility of ST6Gal-I-KD HCT116 cells was caused by the abolition of normal c-Met function, we evaluated HGF-stimulated migration using Boyden chamber assays. In D3 cells, HGF stimulation induced less migration and also eliminated the ability to trigger the development of multicellular-branched structures compared with P and NC cells (Figure 2B). These results indicate that down-regulation of ST6Gal-I expression resulted in c-Met hyposialylation and inhibition of HGFinduced motility of HCT116 cells.

Insufficient terminal $\alpha 2,6$-sialylation abolishes c-Met maturation To elucidate how insufficient terminal a2,6-sialylation determines the fate of the c-Met molecule, we examined c-Met
A

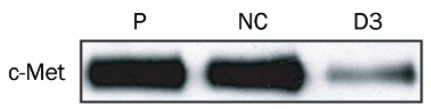

B

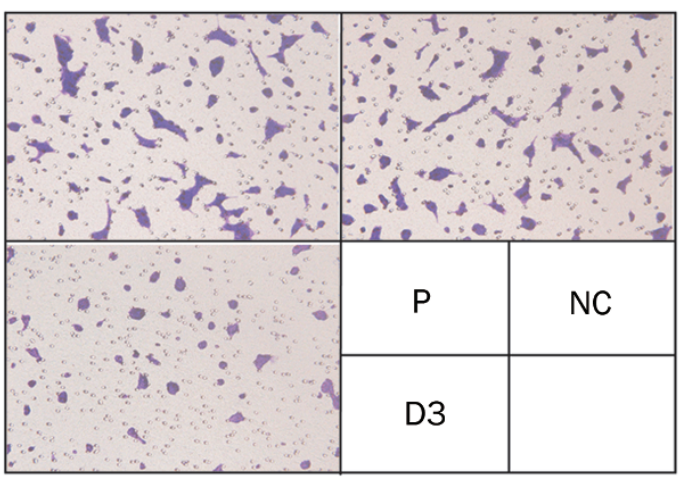

C

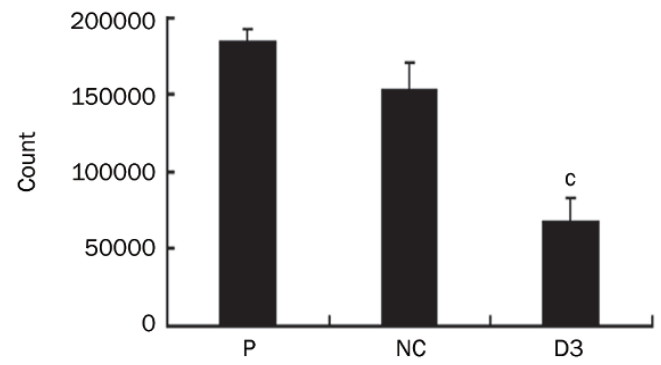

Figure 2. c-Met is hyposialylated in ST6Gal-I-null HCT116 cells. (A) ST6Gal-I knockdown in HCT116 cells caused c-Met hyposialylation. Equivalent amounts of cell lysates were incubated with SNA. SNAglycoprotein complexes were precipitated with streptavidin-conjugated agarose beads and probed by immunoblotting with specific antibody. (B) ST6Gal-I-KD HCT116 cells failed to migrate in response to HGF stimulation. The migration ability of D3, NC, and P cells to HGF stimulation was assessed using a Boyden chamber assay. (C) The migrated cells on the lower surface of the membrane were captured, and five random fields were analyzed using Image-Pro Plus image analysis software. ${ }^{c} P<0.01$ vs NC cells. Columns, means of five independent experiments; bars, SD.

mRNA and protein expression in ST6Gal-I-KD HCT116 cells. Reduced terminal a2,6-sialylation was associated with a decrease in c-Met expression on the surface of D3 cells relative to $\mathrm{P}$ or NC cells (Figure 3A). However, there were no differences in mRNA expression among the three tested cell lines (Figure 3B). These findings indicate that ST6Gal-I affected c-Met function at the post-translational level, rather than at the transcriptional or translational level.

The endoplasmic reticulum (ER)-resident precursor form of c-Met is glycosylated and cleaved into two Golgi-processed mature subunits ( $\alpha$ and $\beta$ ) that are further linked via disulfide bonds into a functional heterodimer on the cell surface. We therefore investigated whether the terminal deficiency in a2,6linkage disrupted c-Met maturation in ST6Gal-I-KD HCT116 cells. Using $\beta$-subunit-specific antibodies, we found that mature $\beta$-subunit was significantly decreased in D3 cells com- 
A
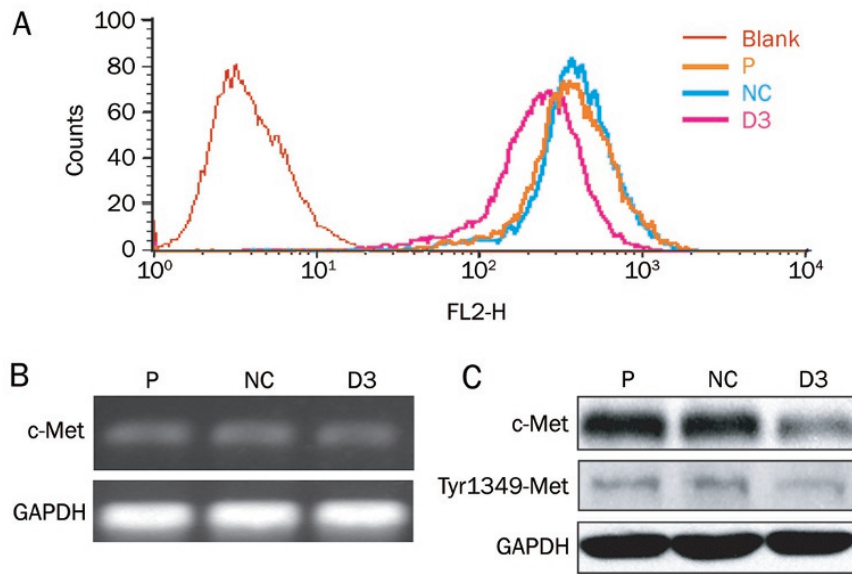

Figure 3. Insufficient terminal $\alpha 2,6$-sialylation abolishes c-Met maturation. (A) ST6Gal-I deficiency affected c-Met expression. c-Met protein expression was analyzed by FACS. (B) ST6Gal-I deficiency had no effect on c-Met transcription examined by RT-PCR. RNA was extracted and amplified as described under Materials and Methods. Glyceraldehyde phosphate 3-dehydrogenase (GAPDH) was used as a control. (C) ST6Gal-I deficiency abolished c-Met maturation. The $\beta$-subunit of c-Met and phosphorylated-c-Met was detected in all cell lines by immunoblotting. GAPDH was used as control.

pared with P or NC cells. Consistently, a reduction in phosphorylated c-Met was noted, mimicking the protein expression profile (Figure 3C).

Tyr705, but not Ser727 phosphorylation of STAT3 responds to hyposialylation in HCT116 cells

We investigated three key downstream targets of c-Met in these transfected HCT116 cells under normal culture conditions. Phosphorylation of STAT3 was down-regulated at Tyr705, rather than Ser727, in ST6Gal-I-null cells, whereas the total level of STAT3 remained unchanged. The other two downstream effectors, Erk1/2 and AKT, were not affected (Figure 4A). Moreover, there was no significant modulation on any of the downstream signaling pathways when cells were starved and stimulated with HGF alone (Figure 4B). These results indicate that insufficient terminal a2,6-sialylation of c-Met or other unidentified glycoproteins directly or indirectly regulate STAT3 Tyr705 dephosphorylation.

\section{Discussion}

Increased levels of ST6Gal-I and a2,6-sialic acid have been observed in various cancer cells and are associated with a poor prognosis in cancer progression ${ }^{[1]]}$. In vitro cell culture studies suggest that ST6Gal-I up-regulation may contribute to metastasis by regulating invasiveness and cell motility. The role of the integrin family members has been emphasized in this context by modifying adhesion to ECM components. However, some studies have reported conflicting results. For example, terminal sialylation reduces attachment to ECM glycoproteins in melanoma ${ }^{[7]}$ and in myeloid cells ${ }^{[8]}$, but enhances adhesion in erythroleukemia K562 cells $^{[9]}$ and colon epithelial SW948
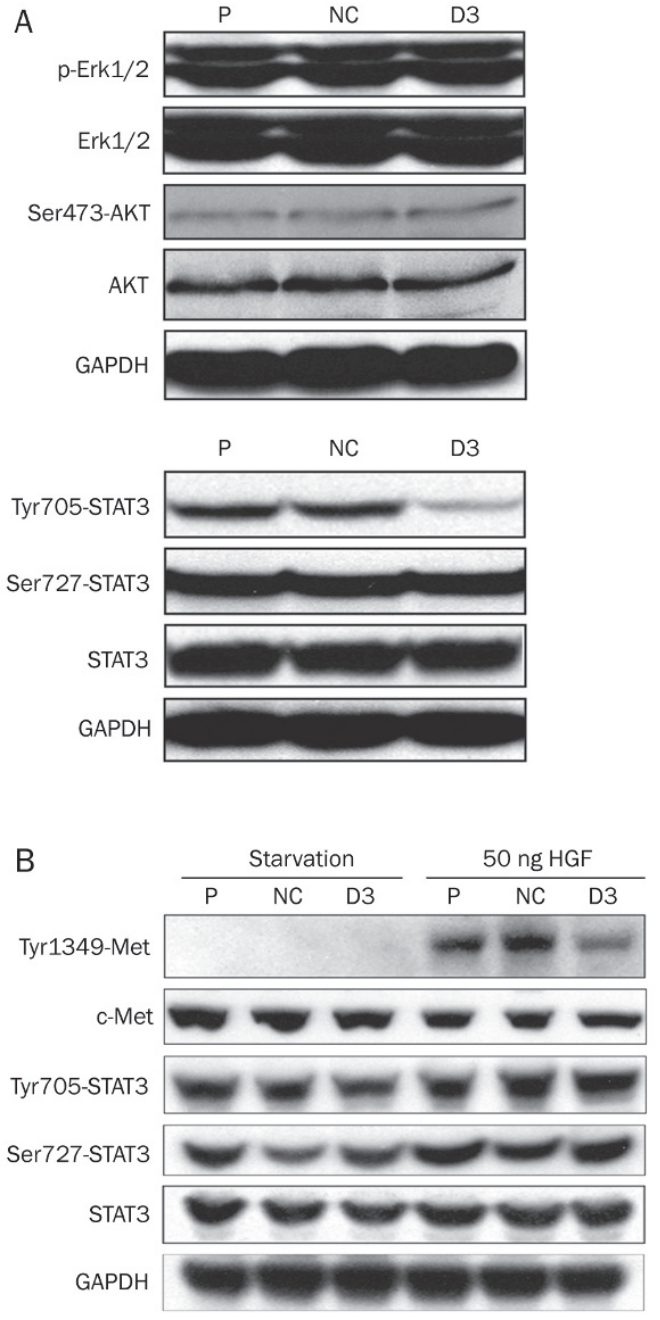

Figure 4. STAT3 tyrosine 705 is sensitive to ST6Gal-I-deficiency in HCT116 cells. (A) STAT3 Tyr705 phosphorylation in ST6Gal-I-deficient HCT116 cells was sensitive to FBS stimulation. Cell lysates were analyzed by Western blotting. After probing for phosphoproteins, the blots were stripped and re-blotted for total proteins to ensure equal loading from $\mathrm{P}$, NC and D3 cell lines. (B) Phosphorylation at the Tyr705 position of STAT3 in ST6Gal-I-deficient HCT116 cells was not sensitive to HGF stimulation. P, $\mathrm{NC}$, and D3 cells were serum-starved for $24 \mathrm{~h}$ and then exposed to $50 \mathrm{ng}$ HGF for 20 min. Cell lysates from starved cells and HGF-stimulated cells were analyzed by Western blotting. The data shown are representative of at least four independent experiments with similar results.

cells ${ }^{[10]}$. These observations support the possibility that integrins are not the only relevant targets of ST6Gal-I-mediated sialylation in cancer cell adhesion and suggest that other surface glycoproteins may also be involved.

Using the siRNA technique in combination with an SNA lectin-blotting approach, we found, for the first time, that ST6Gal-I knock-down specifically abolished HCT116 cell motility via terminal sialylation of c-Met. Despite numerous reports on the glycoprotein nature of c-Met, the potential glycosylation pattern of $\mathrm{N}$-linked carbohydrates on c-Met has not been extensively studied. In our study, ST6Gal-I deficiency 
caused a reduction in a2,6-sialylation of c-Met, supporting the idea that c-Met is the substrate of ST6Gal-I.

Under physiological conditions, c-Met activation is a relatively transient event, whereas in tumor cells, c-Met is often constitutively activated ${ }^{[12]}$. c-Met activation in human tumor cells can be initiated through various mechanisms, including overexpression, structural alterations, and receptor deregulation. The results of the current study suggest that the c-Met hyposialylation is likely to result in long-lasting phenotypic changes. In contrast to the rapid and transient regulatory dynamic characteristics of mature forms of c-Met, the altered glycosylation patterns typical of immature c-Met prevent its efficient trafficking and thus limit its localization to the cell surface. In this context, ST6Gal-I-driven c-Met activation could account for the long-term changes in c-Met-mediated cell motility that are characteristic of neoplastic cells.

Similar to many other growth factor receptor tyrosine kinases, c-Met exerts its oncogenic potential through deregulating the activation of a number of protein phosphorylationdependent signaling cascades ${ }^{[13]}$. These include the Erk/ MAPK and PI3K pathways, which are important for cell adhesion, proliferation, and survival. In the present study, ST6Gal-I null failed to induce adhesion or proliferation. Consistent with this observation, the levels of both total and phosphorylated forms of Erk1/2 and AKT were essentially unchanged following the ST6Gal-I knockdown. Further studies are needed to clarify the biological basis for this lack of effect.

In addition to the Erk/MAPK and PI3K pathways, the activation of c-Met can also induce cell invasion and migration through a complex network of adaptors and transducers. One of these, the transcription factor STAT3, is a key downstream target of c-Met signaling ${ }^{[14]}$. STAT3 is constitutively activated in a number of human tumors and possesses oncogenic potential and anti-apoptotic activities. The precise roles of STAT3 in c-Met signal transduction are not completely understood. Some reports suggested that STAT3 bound directly to c-Met ${ }^{[14]}$ and led to c-Met-dependent formation of branched tubules. Others hold the view that c-Met activates STAT3 indirectly, leading to anchorage-independent growth ${ }^{[15]}$. Regardless of the mechanism, activation by phosphorylation at Tyr705 induces STAT3 dimerization, nuclear translocation and DNA binding ${ }^{[16]}$, whereas phosphorylation at Ser727 is responsible for STAT3 transcriptional activation ${ }^{[17]}$. In the current study, dephosphorylation at Tyr705, but not at Ser727, was observed in ST6Gal-I-KD HCT116 cells upon FBS stimulation. By contrast, in D3 cells, HGF stimulation obviously affects c-Met phosphorylation but has no effect on the phosphorylation status of STAT3 at either Tyr705 or Ser727. These results suggest that a2,6-hyposialylated c-Met could abolish c-Met maturation and destroy its molecular sensitivity to environmental stimuli. The observed dephosphorylation at Tyr705 is likely to be a consequence of a2,6-hyposialylation of all target proteins, rather than being unique to c-Met. The potential involvement of other glycoproteins merits further investigation.

In conclusion, we have shown that c-Met is an additional substrate of ST6Gal-I and that its function is regulated by modification of its a2,6-sialic terminal structure. We also found that elimination of a single terminal sialic acid structure could affect signaling events in HCT116 cells. These findings suggest that therapies targeting toward the ST6Gal-I glycosyltransferase gene may be beneficial, either directly or through synergistic interactions, in treating malignant tumors harboring constitutively activated c-Met.

\section{Acknowledgments}

This work was supported by the National Basic Research Program Grant (№ 2003CB716400) of China, the Natural Science Foundation of China for Distinguished Young Scholars (№ 30725046) and the Natural Science Foundation of China for Innovation Research Group (№ 30721005).

\section{Author contribution}

Prof Mei-yu GENG, Prof Jian DING and Prof Jing LI designed the research. Jin QIAN performed the research, analyzed data and wrote the paper. Cai-hua ZHU, Shuai TANG, Ai-jun SHEN, and Jing AI contributed to the research.

\section{References}

1 Collard JG, Schijven JF, Bikker A, La Riviere G, Bolscher JG, Roos E. Cell surface sialic acid and the invasive and metastatic potential of T-cell hybridomas. Cancer Res 1986; 46: 3521-7.

2 Morgenthaler J, Kemmner W, Brossmer R. Sialic acid dependent cell adhesion to collagen IV correlates with in vivo tumorigenicity of the human colon carcinoma sublines HCT116, HCT116a and HCT116b. Biochem Biophys Res Commun 1990; 171: 860-6.

3 Wang PH, Li YF, Juang CM, Lee YR, Chao HT, Tsai YC, et al. Altered mRNA expression of sialyltransferase in squamous cell carcinomas of the cervix. Gynecol Oncol 2001; 83: 121-7.

4 Seales EC, Jurado GA, Singhal A, Bellis SL. Ras oncogene directs expression of a differentially sialylated, functionally altered beta1 integrin. Oncogene 2003; 22: 7137-45.

5 Seales EC, Jurado GA, Brunson BA, Wakefield JK, Frost AR, Bellis SL. Hypersialylation of beta1 integrins, observed in colon adenocarcinoma, may contribute to cancer progression by up-regulating cell motility. Cancer Res 2005; 65: 4645-52.

6 Lin S, Kemmner W, Grigull S, Schlag PM. Cell surface alpha 2,6 sialylation affects adhesion of breast carcinoma cells. Exp Cell Res 2002; 276: 101-10.

7 Pochec E, Litynska A, Amoresano A, Casbarra A. Glycosylation profile of integrin alpha 3 beta 1 changes with melanoma progression. Biochim Biophys Acta 2003; 1643: 113-23.

8 Semel AC, Seales EC, Singhal A, Eklund EA, Colley KJ, Bellis SL. Hyposialylation of integrins stimulates the activity of myeloid fibronectin receptors. J Biol Chem 2002; 277: 32830-6.

9 Symington BE, Symington FW, Rohrschneider LR. Phorbol ester induces increased expression, altered glycosylation, and reduced adhesion of K562 erythroleukemia cell fibronectin receptors. J Biol Chem 1989; 264: 13258-66.

10 Chiricolo M, Malagolini N, Bonfiglioli S, Dall'Olio F. Phenotypic changes induced by expression of beta-galactoside alpha2,6 sialyltransferase I in the human colon cancer cell line SW948. Glycobiology 2006; 16: 146-54.

11 Lise M, Belluco C, Perera SP, Patel R, Thomas P, Ganguly A. Clinical correlations of alpha2,6-sialyltransferase expression in colorectal 
cancer patients. Hybridoma 2000; 19: 281-6.

12 Takayama H, LaRochelle WJ, Sharp R, Otsuka T, Kriebel P, Anver M, et al. Diverse tumorigenesis associated with aberrant development in mice overexpressing hepatocyte growth factor/scatter factor. Proc Natl Acad Sci USA 1997; 94: 701-6.

13 Blume-Jensen P, Hunter T. Oncogenic kinase signalling. Nature 2001; 411: 355-65.

14 Cramer A, Kleiner S, Westermann M, Meissner A, Lange A, Friedrich K. Activation of the c-Met receptor complex in fibroblasts drives invasive cell behavior by signaling through transcription factor STAT3. J Cell
Biochem 2005; 95: 805-16.

15 Zhang YW, Wang LM, Jove R, Vande Woude GF. Requirement of Stat3 signaling for HGF/SF-Met mediated tumorigenesis. Oncogene 2002; 21: 217-26.

16 Darnell JE Jr, Kerr IM, Stark GR. Jak-STAT pathways and transcriptional activation in response to IFNs and other extracellular signaling proteins. Science 1994; 264: 1415-21.

17 Patten I, Placzek M. Opponent activities of Shh and BMP signaling during floor plate induction in vivo. Curr Biol 2002; 12: 47-52. 